\title{
La gamificación en la educación superior: una revisión sistemática*
}

Carolina Lozada Ávila

Simón Betancur Gómez ${ }^{* * *}$

\author{
Recibido: 29/11/2015 • Aceptado: 21/10/2016 \\ DOI: 10.22395/rium.v16n31a5
}

\begin{abstract}
Resumen
La gamificación se presenta como alternativa a las estrategias tradicionales del aula, y cada vez es más utilizada en la educación superior (ES). El objetivo de la revisión es conocer cómo en estas áreas de conocimiento, la gamificación ha generado mayor interés en términos de producción bibliográfica y uso: administración y economía, arte y humanidades, ciencias de la salud, ciencias naturales y exactas, ciencias sociales y jurídicas, e ingeniería y arquitectura. Se identificó la participación según los resultados en nueve bases de datos académicas. El área con mayor porcentaje, mediante la escala definida en la metodología, es ingeniería y arquitectura $(3,15)$ mientras que ciencias de la salud obtuvo $(0,49)$. Se evidencia que la gamificación es un área explorada en la ES y que son diferentes las experiencias que ha generado su aplicación. Se recomienda establecer lineamientos para su uso, y estudios de mayor alcance para conocer sus verdaderos efectos en el aprendizaje.
\end{abstract}

Palabras clave: área de conocimiento; educación superior; gamificación; juego educativo.

* El artículo es una revisión sistemática, resultado de la asignatura Seminario de Investigación II del posgrado Gerencia de la Información, Facultad de Ingeniería, Universidad de Medellín

** Historiadora. Máster en Gestión documental y administración de archivos. Especialista en Gerencia de la información. Universidad Cooperativa de Colombia Sede Medellín, 3184197092, carolina.lozadaav@ucc.edu.co

*** Ingeniero Industrial. Especialista en Gerencia de la información. Corporación Centro de ciencia y tecnología de Antioquia, sbetancur@cta.org.co 


\title{
Gamification in higher education: a systematic review
}

\begin{abstract}
Gamification is shown as an alternative for traditional classroom strategies and is increasingly used in higher education. The purpose of the revision is to know the way gamification has brought more interest in the following fields of knowledge in terms of bibliographic production and use: management and economics, arts and humanities, health sciences, natural and exact sciences, social and juridical sciences, engineering, and architecture. Participation was identified according to results in nine academic databases. The field with the highest percentage, based on the scale defined in the methodology, is Engineering and Architecture (3.15), while health sciences obtained 0.49. It is clearly seen that gamification is a field explored in higher education and experiences which have generated its application are different. Setting usage guidelines is recommended, including studies of a bigger scope in order to know its real effects on the learning process.
\end{abstract}

Keywords: field of knowledge; higher education; gamification; educational game.

\section{0 gamification no ensino superior: uma revisão sistemática}

\begin{abstract}
Resumo
O gamification apresenta-se como alternativa para as estratégias tradicionais da sala de aula e é cada vez mais utilizado no Ensino Superior (ES). O objetivo desta revisão é conhecer como, nestas áreas de conhecimento, o gamification gera maior interesse em termos de produção bibliográfica e uso: administração e economia, arte e humanas, ciências da saúde, ciências naturais e exatas, ciências sociais e jurídicas, engenharia e arquitetura. Identificou-se a participação segundo os resultados em nove bases de dados acadêmicas. A área com maior porcentagem, mediante a escala definida na metodologia, é engenharia e arquitetura $(3,15)$, enquanto ciências da saúde obteve 0,49. Evidencia-se que o gamification é uma área explorada no ES e que são diferentes as experiências que sua aplicação gera. Recomenda-se estabelecer lineamentos para seu uso e estudos de maior alcance para conhecer seus verdadeiros efeitos na aprendizagem.
\end{abstract}

Palavras-chave: área de conhecimento; ensino superior; gamification; jogo educativo. 


\section{INTRODUCCIÓN}

La necesidad constante de actualizar los métodos educativos debe ser considerada en pro de mejorar la calidad de la educación, que depende principalmente de los contenidos que se imparten, las necesidades de la sociedad y la cobertura. Por ello, cada vez es más frecuente recurrir a elementos como las TIC y aplicaciones lúdicas que apoyen el proceso [1].

Desde el enfoque de Durán Rodríguez [2], la enseñanza aprendizaje define al profesor como el eje del proceso en el aula, lo que es considerado por muchos docentes en la actualidad como poco productivo y mecánico, dado que el alumno se esfuerza poco y pueden verse afectadas la formación de valores y la adquisición de normas de comportamiento y de métodos de aprendizaje. Por lo anterior, se debe reflexionar sobre estos métodos buscando mayor interacción del estudiante y, como fin último, cumplir con los propósitos formativos asociados a la preparación profesional y a la formación de competencias para una integración positiva a la sociedad del conocimiento [2].

En la educación, la gamificación está ganando un importante lugar, siendo empleada como técnica para motivar a los estudiantes en su proceso de aprendizaje [3]. El juego es un activador en la atención y surge como alternativa para complementar los esquemas de enseñanza tradicional. Esto último motivó el interés de conocer cómo la gamificación ha sido empleada en la Educación Superior. De igual forma, se identificó que para conocer su uso en dicho ámbito se requiere un abordaje según los diferentes campos de conocimiento que agrupan los saberes, y que son los que definen los programas que se imparten en las instituciones.

Por lo tanto, el objetivo de la revisión es identificar, mediantes casos aplicados en las diferentes áreas del conocimiento, cómo la gamificación ha sido empleada en la Educación Superior, así como también conocer mediante cadenas de búsqueda, su participación en la literatura.

Para lograr lo propuesto, se presenta un marco conceptual y el detalle del método empleado. Posteriormente, se muestran los resultados obtenidos en la revisión, identificando cada una de las áreas de conocimiento y el nivel de recurrencia según las cadenas de búsqueda. Se presentan los trabajos seleccionados y las brechas identificadas en los mismos. Finalmente, como solución se enumera una serie de recomendaciones y perspectivas para el futuro de la gamificación en la Educación Superior.

\section{MARCO CONCEPTUAL}

De acuerdo con el objetivo de esta revisión, se reconoce la importancia de ampliar el concepto de gamificación y cuáles son los elementos que, según algunos autores, se 
debe tener para su aplicación. Para conocer el uso en la Educación Superior, se abordará desde las áreas de conocimiento que se imparten en el ámbito académico, y se define qué se entiende por este concepto y cómo será trabajado en el desarrollo de la revisión.

\section{Gamificación}

A partir de los trabajos revisados, se puede establecer que la gamificación no cuenta con una definición universal o un marco común. Una manera muy simple de entenderla es la utilización del juego en contextos diferentes a este. Tal como lo definen Richard N. Landers y Rachel C. Callan [3] "la adición de elementos comúnmente asociados con los juegos". Por otro lado, se encuentra un concepto donde el objetivo se entiende como "el uso de la mecánica del juego, dinámica y los marcos para promover comportamientos deseados" [4]. Según este concepto y como abordan el tema en su trabajo, el promover se entiende como la motivación para lograr algo; en este caso, motivar el aprendizaje. Los autores Joey J. Lee y Jessica Hammer, identifican que la gamificación es una oportunidad para enfrentar dos problemas en la educación: la motivación y el compromiso [4] y que puede servir como apoyo, especialmente en tres áreas: a) Cognoscitiva: En estas se identifican las reglas de juego que el jugador encuentra con la exploración activa y el descubrimiento. Plantean objetivos medianamente difíciles que motivan alcanzarlos y de los cuales obtienen recompensas inmediatas. Además, llevan al éxito. b) Emocional: Los juegos invocan variedad de emociones, experiencias emocionalmente positivas y de orgullo, y también pueden lograr ver el fracaso como una oportunidad, en vez de percibirlo como indefensión, temor o sensación de ser dominado. c) Social: el juego permite que se prueben nuevas identidades y papeles, requiriendo que tomen decisiones ante sus nuevas posiciones. Asimismo, ayuda a que el jugador asuma un rol activo del cual obtenga buenos resultados para el aprendizaje.

\section{Juego}

Para que sea posible cumplir objetivos de aprendizaje, el juego debe adquirir unas características particulares en términos de aspectos a considerar en su diseño y, dado que es intencional, debe contar con un proceso sistémico de desarrollo que Marne, Wisdom, et al. [5] definen en seis aspectos:

a) Objetivo pedagógico: definir el alcance y representarlo a través de modelos que indiquen el dominio de conocimiento. Dicha modelación debe ser construida en conjunto con los expertos pedagógicos dado que estos son los que aportan el contexto.

b) Simulación: de modo que sea un juego, este debe tener reglas y parámetros establecidos claramente que permitan que este se replique y no genere situaciones 
interpretativas que no se encuentren contempladas. Al igual que el objetivo pedagógico, este aspecto requiere ser trabajado en conjunto con los expertos.

c) Interacción con la simulación: este aspecto ahonda en la manera en la cual el jugador interactúa, de modo que esta lleve al aprendizaje. El trabajo de expertos en juegos es requerido para ello.

d) Problemas y progresión: Puntualizada la interacción, se debe desarrollar la ruta metodológica que colocará la serie de desafíos de aprendizaje en el orden indicado para el cumplimiento del objetivo. Además de definir la ruta, se debe especificar la realimentación que se otorga al jugador acerca de su progreso.

e) Decoración: Precisar qué objetos multimedia se utilizarán para atraer la atención del jugador; estos son elementos no relacionados con el objetivo de conocimiento que agregan otros aspectos y enriquecen la experiencia de usuario.

f) Condición de uso: Delimitar quién, cuándo, dónde y cómo se utilizará el juego. Se debe indicar el contexto en el cual el juego cumple su objetivo y las reglas de ese contexto. Así, un juego podrá ser virtual, asincrónico, grupal, individual, entre otros, pero siempre cumpliendo con su objetivo de aprendizaje.

\section{Áreas de conocimiento}

En Colombia, las áreas de conocimiento son definidas por el Ministerio de Educación Nacional como “agrupación que se hace de los programas académicos, teniendo en cuenta cierta afinidad en los contenidos, en los campos específicos del conocimiento, en los campos de acción de la educación superior cuyos propósitos de formación conduzcan a la investigación o al desempeño de ocupaciones, profesiones y disciplinas" [6]. Para esta revisión se define trabajar solo con 6 agrupaciones, con el interés de lograr más efectividad en la búsqueda, teniendo en cuenta los diferentes contextos en los que se puedan encontrar trabajos y coincidan con estas áreas. A continuación, se detallan las áreas y las disciplinas asociadas:

- Administración y economía (Contabilidad, Economía, Administración)

- Arte y humanidades (Música, Teatro, Artes)

- Ciencias de la salud (Enfermería, Medicina, Farmacia)

- Ciencias naturales y exactas (Astronomía Biología, Física, Matemáticas y afines, Geología, Química)

- Ciencias sociales y jurídicas (Derecho, Antropología, Historia, Geografía, Sociología; Psicología)

- Ingeniería y arquitectura (Ingenierías, Arquitectura) 


\section{MÉTODO}

El desarrollo de la revisión sistemática se hace en 3 etapas: planeación, búsqueda y documentación.

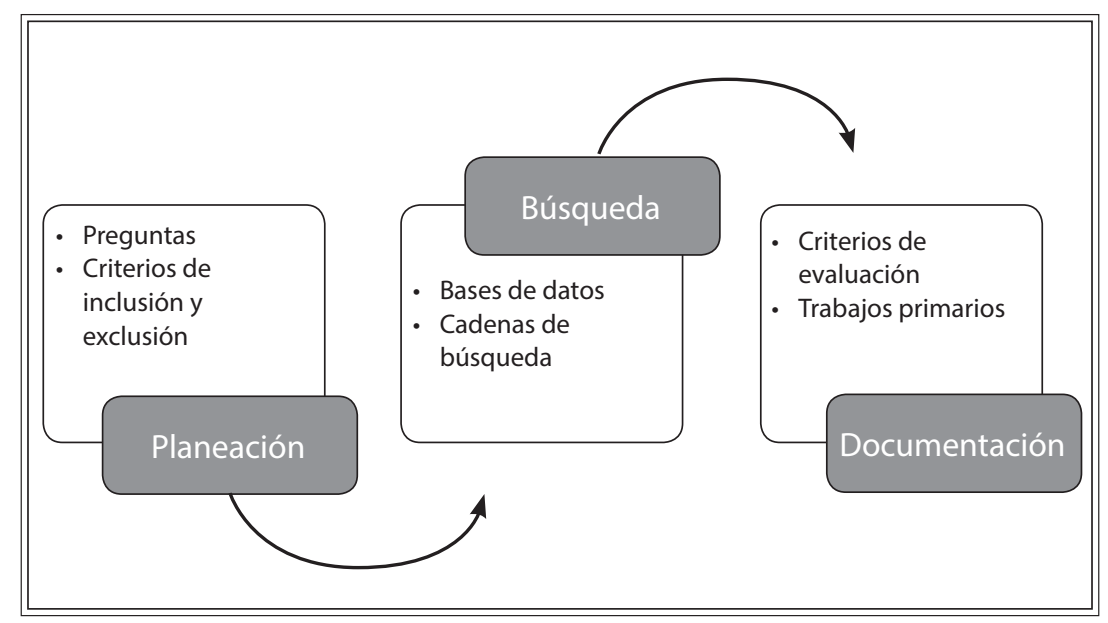

Figura 1. Etapas del método aplicado a la revisión sistemática Fuente: [7]

Al iniciar el proceso se hace la lectura y conceptualización general sobre la gamificación, sus aplicaciones y desarrollo. Posteriormente, se decidió profundizar sobre esta metodología en la Educación Superior, no solo para delimitar el tema y hacer la revisión más específica, sino porque se identificó que ha sido muy poco estudiada.

\section{Planeación}

El propósito de la búsqueda se orienta hacia un conjunto de trabajos relacionados con experiencias de gamificación en la Educación Superior en las 6 áreas de conocimiento de interés, con el fin de determinar el uso que se le ha dado a esta metodología y su participación en las áreas elegidas y sus disciplinas.

Para esta revisión se definieron las siguientes preguntas:

Pregunta Central: de acuerdo con el rastreo de estudios sobre el tema, ¿en qué área de conocimiento está teniendo mayor participación la gamificación en la Educación Superior?

Preguntas complementarias: ¿Cómo se está abordando la gamificación en la Educación Superior en las diferentes áreas de conocimiento?

¿En qué áreas de conocimiento la gamificación presenta menor participación? 
Para la selección de los estudios se tienen en cuenta los siguientes criterios de inclusión y exclusión, los cuales son identificados según las preguntas y el objeto de la revisión:

\section{Criterios de inclusión}

- Trabajos publicados del año 2000 en adelante

- Búsqueda en bases de datos académicas como: Ebsco, ACM, IEEE, springer link, Web of Science, Emerald, Science Direct, Scopus, Google Scholar.

- Publicaciones como artículos de revista, libros, capítulos de libros, presentaciones en eventos académicos que trabajen la gamificación en Educación Superior.

- Todos los contextos geográficos

- Trabajos que hayan implementado la gamificación en las áreas de conocimiento definidas en la revisión.

\section{Criterios de exclusión}

- Trabajos que no procedan de fuentes académicas confiables

- Publicaciones que trabajen la gamificación en Educación Básica y Media u otro tipo de ambientes académicos distintos a la Educación Superior.

- Referencias anteriores al 2000

- Artículos que estudien la gamificación en organizaciones, manejo de clientes, capacitaciones, etc.

\section{Búsqueda}

Se realiza la consulta en las bases de datos académicas seleccionadas, atendiendo al objetivo principal de la revisión. Para ello, se tomaron en cuenta palabras clave, tipos de publicación y las áreas de conocimiento con sus disciplinas.

La primera cadena de búsqueda es de carácter general, con el objetivo de hacer una exploración de los resultados de las palabras clave: gamificación y Educación Superior.

\section{General: gamification AND higher education}

La segunda cadena se precisa agregando el área de conocimiento a la cadena general, para obtener el número de trabajos que se han desarrollado en cada área. Esto para detallar más la búsqueda y, asimismo, poder realizar con el número de registros obtenidos, un análisis de similitudes o diferencias frente a la literatura sobre gamificación 
en la Educación Superior, dependiendo del área.

\section{Ejemplo: Gamification AND Higher education AND Biology}

La tabla 1 muestra el detalle de la cadena de búsqueda con cada una de las áreas de conocimiento y su composición, según a las disciplinas que fueron definidas en la revisión.

Tabla 1. Ecuaciones de búsqueda utilizadas

\begin{tabular}{|c|c|}
\hline General & Gamification AND higher education \\
\hline Ciencias naturales y Exactas & $\begin{array}{l}\text { Gamification AND Higher education AND Natural sciences; Gamification } \\
\text { AND Higher education AND Astronomy; Gamification AND Higher } \\
\text { education AND Biology; Gamification AND Higher education AND } \\
\text { physics; Gamification AND Higher education AND Mathematics; Ga- } \\
\text { mification AND Higher education AND Geology; Gamification AND } \\
\text { Higher education AND Chemistry }\end{array}$ \\
\hline Ciencias Sociales y Jurídicas & $\begin{array}{l}\text { Gamification AND Higher education AND Social sciences; Gamifi- } \\
\text { cation AND Higher education AND Law; Gamification AND Higher } \\
\text { education AND Antropology; Gamification AND Higher education } \\
\text { AND History; Gamification AND Higher education AND Geography; } \\
\text { Gamification AND Higher education AND Sociology; Gamification } \\
\text { AND Higher education AND psychology }\end{array}$ \\
\hline Ciencias de la Salud & $\begin{array}{l}\text { Gamification AND Higher education AND Health sciences; Gami- } \\
\text { fication AND Higher education AND Nursery; Gamification AND } \\
\text { Higher education AND Medical; Gamification AND Higher education } \\
\text { AND Pharmacy }\end{array}$ \\
\hline Ingeniería y arquitectura & $\begin{array}{l}\text { Gamification AND higher education AND engineering; gamification } \\
\text { AND higher education AND architecture }\end{array}$ \\
\hline Administración y economía & $\begin{array}{l}\text { Gamification AND higher education AND management science; } \\
\text { gamification AND higher education AND accounting; gamification } \\
\text { AND higher education AND management; gamification AND higher } \\
\text { education AND economy; gamification AND higher education AND } \\
\text { administration }\end{array}$ \\
\hline Artes y Humanidades & $\begin{array}{l}\text { Gamification AND higher education AND humanities; gamification } \\
\text { AND higher education AND music; gamification AND higher education } \\
\text { AND theatre; gamification AND higher education AND Arts }\end{array}$ \\
\hline
\end{tabular}

Fuente: elaboración autores

\section{Documentación}

Se concretan cuatro criterios de evaluación con la siguiente escala de valoración Alto 
(5), Medio (3), Bajo (1) de acuerdo con la pertinencia de estos aspectos en los artículos, y a partir de estos, seleccionar los estudios primarios que orientarán el análisis y ayudarán a dar respuesta a las preguntas formuladas:

- Aplicación de la gamificación (C01): Este criterio permite identificar cómo fue empleada la gamificación por parte del autor, bajo qué metodología y qué tanto se ajusta al concepto y lo contemplado por este término. Resultado obtenido en el desarrollo de la temática.

- Coherencia metodológica (C02): califica la metodología utilizada en el estudio. Esta debe ser clara, con alcance determinado y concluyente.

- Claridad de los argumentos (C03): Califica la claridad de los argumentos presentados por el autor, teniendo en cuenta los resultados obtenidos.

- Aporte al área de conocimiento (C04): Con este criterio se busca identificar cómo aporta el trabajo al área de conocimiento sobre el uso de la gamificación identificando una proyección futura de aplicabilidad.

En la tabla 2, se relacionan los artículos elegidos para el estudio, de acuerdo con la pertinencia y el cumplimiento de la evaluación de los criterios; en la tabla 3 se relacionan los artículos elegidos como estudios primarios y las veces que han sido citados por otros autores. Y en la tabla 4 se presentan la síntesis de los trabajos rastreados en la revisión.

Tabla 2. Artículos evaluados

\begin{tabular}{|c|l|l|l|c|c|c|c|c|c|}
\hline ID & $\begin{array}{l}\text { Área de co- } \\
\text { nocimiento }\end{array}$ & Título & Autores & Año & CO1 & CO2 & CO3 & CO4 & Total \\
\hline 1 & $\begin{array}{l}\text { Ciencias } \\
\text { Sociales y } \\
\text { Jurídicas }\end{array}$ & $\begin{array}{l}\text { ARG (Alternate Reality Games) } \\
\text { Contributions, Limitations, and } \\
\text { Potentialities to the Service of } \\
\text { the Teaching at the University } \\
\text { Level }\end{array}$ & $\begin{array}{l}\text { T Pineiro- } \\
\text { Otero, C } \\
\text { Costa-Sán- } \\
\text { chez }\end{array}$ & 2015 & 3 & 3 & 3 & 1 & $50 \%$ \\
\hline 2 & $\begin{array}{l}\text { Ciencias } \\
\text { Naturales y } \\
\text { exactas }\end{array}$ & $\begin{array}{l}\text { How Gamification Applies for } \\
\text { Educational Purpose Specially } \\
\text { with College Algebra }\end{array}$ & $\begin{array}{l}\text { U Faghihi, } \\
\text { A Brau- } \\
\text { tigam, K } \\
\text { Jorgenson, } \\
\text { D Martin }\end{array}$ & 2014 & 5 & 5 & 3 & 5 & $90 \%$ \\
\hline
\end{tabular}




\begin{tabular}{|c|c|c|c|c|c|c|c|c|c|}
\hline$I D$ & $\begin{array}{l}\text { Área de co- } \\
\text { nocimiento }\end{array}$ & Título & Autores & Año & $\mathrm{CO}$ & $\mathrm{CO} 2$ & $\mathrm{CO} 3$ & $\mathrm{CO} 4$ & Total \\
\hline 3 & $\begin{array}{l}\text { Ciencias } \\
\text { Sociales y } \\
\text { Jurídicas }\end{array}$ & $\begin{array}{l}\text { Casual Social Games as Serious } \\
\text { Games: The Psychology of } \\
\text { Gamification in Undergraduate } \\
\text { Education and Employee } \\
\text { Training }\end{array}$ & $\begin{array}{l}\text { RN Landers, } \\
\text { RC Callan }\end{array}$ & 2011 & 5 & 3 & 3 & 5 & $80 \%$ \\
\hline 4 & $\begin{array}{l}\text { Ciencia } \\
\text { Naturales y } \\
\text { exactas }\end{array}$ & $\begin{array}{l}\text { Improving biotech education } \\
\text { through gamified laboratory } \\
\text { simulations }\end{array}$ & $\begin{array}{l}\text { MT Bonde, } \\
\text { G Makrans- } \\
\text { ky, J Wan- } \\
\text { dall, MV } \\
\text { Larsen }\end{array}$ & 2014 & 5 & 3 & 5 & 5 & $90 \%$ \\
\hline 5 & $\begin{array}{l}\text { Ciencias } \\
\text { Sociales y } \\
\text { Jurídicas }\end{array}$ & $\begin{array}{l}\text { APRENDER JUGANDO: } \\
\text { Experiencias de aprendizaje } \\
\text { mediante juegos en la Facultad } \\
\text { de Derecho de la UdG }\end{array}$ & $\begin{array}{l}\text { A Ruda } \\
\text { González }\end{array}$ & 2014 & 5 & 3 & 3 & 5 & $80 \%$ \\
\hline 6 & $\begin{array}{l}\text { Ciencias de } \\
\text { la salud }\end{array}$ & $\begin{array}{l}\text { Designing Serious Games for } \\
\text { Learning Support in Medicine } \\
\text { Studies: A Specific Method } \\
\text { to Elicit and Formalize } \\
\text { Requirements }\end{array}$ & $\begin{array}{l}\text { O Colteli, } \\
\text { X Grandi, } \\
\text { R Tosca, P } \\
\text { Latorre }\end{array}$ & 2014 & 1 & 5 & 5 & 5 & $80 \%$ \\
\hline 7 & $\begin{array}{l}\text { Ciencias de } \\
\text { la salud }\end{array}$ & $\begin{array}{l}\text { Futuro de la enseñanza médica: } \\
\text { inteligencia artificial y big data }\end{array}$ & $\begin{array}{l}\text { Ó García } \\
\text { Esquirol }\end{array}$ & 2015 & 5 & 1 & 3 & 3 & $60 \%$ \\
\hline 8 & $\begin{array}{l}\text { Ciencias de } \\
\text { la salud }\end{array}$ & $\begin{array}{l}\text { Gamification as a Learning } \\
\text { Method in Pharmacy Education }\end{array}$ & $\begin{array}{l}\text { MS } \\
\text { Shawaqfeh }\end{array}$ & 2015 & 3 & 5 & 5 & 5 & $90 \%$ \\
\hline 9 & $\begin{array}{l}\text { Ciencias de } \\
\text { la salud }\end{array}$ & $\begin{array}{l}\text { Gamification as a tool for } \\
\text { enhancing graduate medical } \\
\text { education }\end{array}$ & $\begin{array}{l}\text { CR Nevin, } \\
\text { AO West- } \\
\text { fall, JM } \\
\text { Rodriguez }\end{array}$ & 2014 & 5 & 5 & 3 & 3 & $80 \%$ \\
\hline 10 & $\begin{array}{l}\text { Ciencias de } \\
\text { la salud }\end{array}$ & $\begin{array}{l}\text { Gamification for Internet Based } \\
\text { Learning in Health Professions } \\
\text { Education. }\end{array}$ & $\begin{array}{l}\text { D Rojas, B } \\
\text { Kapralos }\end{array}$ & 2014 & 1 & 3 & 5 & 5 & $70 \%$ \\
\hline 11 & $\begin{array}{l}\text { Inge- } \\
\text { niería y } \\
\text { arquitectura }\end{array}$ & $\begin{array}{l}\text { Gamification and Rule } \\
\text { Based Design Strategies in } \\
\text { Architecture Education }\end{array}$ & $\begin{array}{l}\text { MA } \\
\text { Schnabel, } \\
\text { SLT Tian, S } \\
\text { Aydin }\end{array}$ & 2014 & 5 & 3 & 3 & 3 & $70 \%$ \\
\hline 12 & $\begin{array}{l}\text { Inge- } \\
\text { niería y } \\
\text { arquitectura }\end{array}$ & $\begin{array}{l}\text { Gamification for engaging } \\
\text { computer science students } \\
\text { in learning activities: A case } \\
\text { study }\end{array}$ & $\begin{array}{l}\text { MB Ibanez, } \\
\text { A Di-Serio }\end{array}$ & 2014 & 5 & 5 & 3 & 3 & $80 \%$ \\
\hline
\end{tabular}




\begin{tabular}{|c|c|c|c|c|c|c|c|c|c|}
\hline$I D$ & $\begin{array}{l}\text { Área de co- } \\
\text { nocimiento }\end{array}$ & Título & Autores & $A \tilde{n} o$ & $\mathrm{COI}$ & $\mathrm{CO} 2$ & $\mathrm{CO} 3$ & $\mathrm{CO} 4$ & Total \\
\hline 13 & $\begin{array}{l}\text { Inge- } \\
\text { niería y } \\
\text { arquitectura }\end{array}$ & $\begin{array}{l}\text { Engaging Engineering Students } \\
\text { with Gamification }\end{array}$ & $\begin{array}{l}\text { G Barata, } \\
\text { S Gama, J } \\
\text { Jorge }\end{array}$ & 2013 & 5 & 5 & 3 & 3 & $80 \%$ \\
\hline 14 & $\begin{array}{l}\text { Inge- } \\
\text { niería y } \\
\text { arquitectura }\end{array}$ & $\begin{array}{l}\text { Panorámica: serious games, } \\
\text { gamificación y mucho más }\end{array}$ & $\begin{array}{l}\text { FJ Gallego, } \\
\text { CJ Villagrá, } \\
\text { R Satorre }\end{array}$ & 2014 & 5 & 3 & 3 & 1 & $60 \%$ \\
\hline 15 & $\begin{array}{l}\text { Adminis- } \\
\text { tración y } \\
\text { economía }\end{array}$ & $\begin{array}{l}\text { Experiencias de aprendizaje } \\
\text { significativo mediante } \\
\text { ludificación en un curso de } \\
\text { administración }\end{array}$ & $\begin{array}{l}\text { EB Rangel, } \\
\text { FM del } \\
\text { Campo Se- } \\
\text { púlveda, GI } \\
\text { Castanedo }\end{array}$ & 2014 & 5 & 5 & 5 & 3 & $90 \%$ \\
\hline 16 & $\begin{array}{l}\text { Ciencias } \\
\text { Sociales y } \\
\text { Jurídicas }\end{array}$ & $\begin{array}{l}\text { Assessing the effects of } \\
\text { gamification in the classroom: } \\
\text { A longitudinal study on } \\
\text { intrinsic otivation, social } \\
\text { comparison, satisfaction, effort, } \\
\text { and academic performance }\end{array}$ & $\begin{array}{l}\text { MD Hanus, } \\
\text { J Fox }\end{array}$ & 2015 & 5 & 5 & 3 & 5 & $90 \%$ \\
\hline 17 & $\begin{array}{l}\text { Inge- } \\
\text { niería y } \\
\text { arquitectura }\end{array}$ & $\begin{array}{l}\text { Implementing gamification } \\
\text { techniques into university study } \\
\text { path - a case study }\end{array}$ & $\begin{array}{l}\text { M } \\
\text { Laskowski }\end{array}$ & 2015 & 3 & 5 & 5 & 5 & $90 \%$ \\
\hline 18 & $\begin{array}{l}\text { Artes y hu- } \\
\text { manidades }\end{array}$ & $\begin{array}{l}\text { Gamification for Learning 3D } \\
\text { Computer Graphics Arts }\end{array}$ & $\begin{array}{l}\text { S Villagra- } \\
\text { sa, J Duran }\end{array}$ & 2013 & 5 & 3 & 3 & 3 & $70 \%$ \\
\hline 19 & $\begin{array}{l}\text { Artes y } \\
\text { humanida- } \\
\text { des }\end{array}$ & $\begin{array}{l}\text { Virtual worlds in Australian } \\
\text { and New Zealand higher } \\
\text { education: Remembering the } \\
\text { past, understanding the present } \\
\text { and imagining the future }\end{array}$ & $\begin{array}{l}\text { S Gregory, } \\
\text { B Gregory, } \\
\text { T Reiners, A } \\
\text { Fardinpour }\end{array}$ & 2013 & 1 & 5 & 3 & 3 & $60 \%$ \\
\hline
\end{tabular}

Fuente: elaboración autores

Tabla 3. Estudios Primarios

\begin{tabular}{|c|l|l|l|l|c|c|}
\hline ID & \multicolumn{1}{|c|}{$\begin{array}{c}\text { Área de } \\
\text { conocimiento }\end{array}$} & \multicolumn{1}{|c|}{ Título } & Autores & País & Año & $\begin{array}{c}\text { Cita- } \\
\text { ciones }\end{array}$ \\
\hline \multirow{4}{*}{15} & $\begin{array}{l}\text { Adminis- } \\
\text { tración y } \\
\text { economía }\end{array}$ & $\begin{array}{l}\text { Experiencias de aprendi- } \\
\text { zaje significativo mediante } \\
\text { ludificación en un curso de } \\
\text { administración }\end{array}$ & $\begin{array}{l}\text { Enrique Bores Rangel; } \\
\text { Fernando Martín del } \\
\text { Campo Sepúlveda; Ga- } \\
\text { briela Ibarra Castanedo }\end{array}$ & España & 2014 & 0 \\
\hline \multirow{4}{*}{$\begin{array}{l}\text { Ciencia } \\
\text { Naturales y } \\
\text { exactas }\end{array}$} & $\begin{array}{l}\text { Improving biotech education } \\
\text { through gamified laboratory } \\
\text { simulations }\end{array}$ & $\begin{array}{l}\text { Bonde, M. T., Makransky, } \\
\text { G.,Wandall, J.,Larsen, M. } \\
\text { V., Morsing, M., Jarmer, } \\
\text { H., \& Sommer, M. O }\end{array}$ & Dinamarca & 2014 & 3 \\
\hline
\end{tabular}




\begin{tabular}{|c|c|c|c|c|c|c|}
\hline$I D$ & $\begin{array}{c}\text { Área de } \\
\text { conocimiento }\end{array}$ & Título & Autores & Pais & Año & $\begin{array}{l}\text { Cita- } \\
\text { ciones }\end{array}$ \\
\hline 8 & $\begin{array}{l}\text { Ciencias de } \\
\text { la salud }\end{array}$ & $\begin{array}{l}\text { Gamification as a Lear- } \\
\text { ning Method in Pharmacy } \\
\text { Education }\end{array}$ & Mohammad S Shawaqfeh & $\begin{array}{l}\text { Estados } \\
\text { Unidos }\end{array}$ & 2015 & 0 \\
\hline 16 & $\begin{array}{l}\text { Ciencias } \\
\text { Sociales y } \\
\text { Jurídicas }\end{array}$ & $\begin{array}{l}\text { Assessing the effects of gami- } \\
\text { fication in the classroom: A } \\
\text { longitudinal study on intrin- } \\
\text { sic motivation, social compa- } \\
\text { rison, satisfaction, effort, and } \\
\text { academic performance }\end{array}$ & $\begin{array}{l}\text { Michael D Hanus, Jesse } \\
\text { Fox }\end{array}$ & $\begin{array}{l}\text { Estados } \\
\text { Unidos }\end{array}$ & 2015 & 109 \\
\hline 17 & $\begin{array}{l}\text { Ingeniería y } \\
\text { arquitectura }\end{array}$ & $\begin{array}{l}\text { Implementing gamification } \\
\text { techniques into university } \\
\text { study path - a case study }\end{array}$ & Maciej Laskowski & Polonia & 2015 & 3 \\
\hline 18 & $\begin{array}{l}\text { Artes y } \\
\text { humanidades }\end{array}$ & $\begin{array}{l}\text { Gamification for Learning } \\
\text { 3D Computer Graphics Arts }\end{array}$ & $\begin{array}{l}\text { Sergi Villagrasa, Jaume } \\
\text { Duran }\end{array}$ & España & 2013 & 14 \\
\hline
\end{tabular}

Fuente: elaboración autores

Tabla 4. Síntesis de artículos

\begin{tabular}{|c|l|}
\hline$I D$ & \multicolumn{1}{c|}{ Síntesis del artículo } \\
\hline 1 & $\begin{array}{l}\text { El artículo hace una aproximación en el uso de las ARG (Juegos de Realidad Alternativa) en la } \\
\text { Educación superior, reconociendo las bondades que estos juegos dan en otras áreas como el mar- } \\
\text { keting. El objetivo es identificar cómo se han implementado en la Educación. Las ARG, permiten } \\
\text { estrategias de integración características de juegos como de software social, combinando lo real } \\
\text { y lo virtual. Los juegos le permiten ser sus propios conductores y hacer uso de sus experiencias y } \\
\text { conocimientos y avanzar en el juego. Se propone esta alternativa, tomando como base la afirmación } \\
\text { de que la educación debe estar acorde con los contextos tecnológicos de los estudiantes y dando } \\
\text { la oportunidad de que estos empleen sus conocimientos y los desarrollen por medio del juego. }\end{array}$ \\
\hline 2 & $\begin{array}{l}\text { Este artículo muestra el uso de un sistema (MathDungeon) para la enseñanza de las matemáticas a } \\
\text { nivel Universitario. La propuesta del sistema, incluye lecciones, animación artística, música,juegos } \\
\text { para cada concepto, ejemplos, resolución de problemas, puntuación y humor, logrando retener } \\
\text { aprendizaje. Para probar el sistema de tutoría matemática, se hizo una prueba con 30 estudiantes } \\
\text { de la Universidad Estatal Sul Ross (SRSU). Se concluye que las herramientas de gamificación, } \\
\text { pueden incrementar la confianza en el aprendizaje. }\end{array}$ \\
\hline 3 & $\begin{array}{l}\text { En este capítulo se analiza, como la gamificación puede ser un método adecuado para transferir } \\
\text { conocimiento orientado a los resultados. El estudio se realiza con } 600 \text { estudiantes de pregrado } \\
\text { del departamento de Psicología de la Universidad de la Costa Este, donde hay la oportunidad de } \\
\text { interactuar en un sitio de red social (SNS). Se obtuvo importantes resultados de alto interés en la } \\
\text { red social por parte de los estudiantes. Considera que el éxito de la gamificación, la orientación al } \\
\text { resultado, las motivaciones de aprendizaje para ganar algo como una recompensa por aprender. }\end{array}$ \\
\hline
\end{tabular}




\begin{tabular}{|c|c|}
\hline$I D$ & Sintesis del artículo \\
\hline 4 & $\begin{array}{l}\text { Este artículo analiza la enseñanza en el área de las ciencias, especialmente la docencia en los } \\
\text { laboratorios. Se reconoce la tecnología, como una oportunidad de afianzar y motivar a los estu- } \\
\text { diantes. En un estudio en Dinamarca, un porcentaje importante de estudiantes afirmo contemplar } \\
\text { la posibilidad de estudiar carreras de ciencias luego de utilizar simuladores de laboratorios. Los } \\
\text { simuladores están dando significativas posibilidades de aprendizaje a los estudiantes mostrando } \\
\text { un avance del } 76 \% \text { de crecimiento en el aprendizaje. }\end{array}$ \\
\hline 5 & $\begin{array}{l}\text { Este artículo expone una iniciativa con gamificación en la Facultad de Derecho de la Universidad } \\
\text { de la Universitat de Girona. Esto se ha desarrollado en el curso Historia del Derecho con juegos } \\
\text { como: Maquiavelli(Italia renacentista) y Timeline (cronologías históricas). Con el avance de esta } \\
\text { metodologia en algunas asignaturas, se generó la iniciativa de crear una ludoteca en la Facultad) } \\
\text { de Derecho, La "UdGLudoteca", motivando a los estudiantes y aportando también a los profesores } \\
\text { con material de curso. Con la ludoteca se ha generado mayor interés en este método de aprendizaje } \\
\text { que está dando importantes cambios en la enseñanza del Derecho. }\end{array}$ \\
\hline 6 & $\begin{array}{l}\text { Este es un artículo acerca de los avances de un trabajo que continúa desarrollándose. Las facultades } \\
\text { de medicina en España cada vez realizan un uso más intensivo de las TIC para apoyar el proceso } \\
\text { de enseñanza aprendizaje y la gamificación muestra ser un recurso que viabiliza esta iniciativa, } \\
\text { no obstante la carencia de metodologías ajustadas al área de la salud genera una brecha que debe } \\
\text { ser atendida con el fin de intensionar aún más el proceso de creación de contenidos educativos } \\
\text { mediante la gamificación. Este artículo propone un abordaje del establecimiento de requerimientos } \\
\text { para maximizar esta intencionalidad. }\end{array}$ \\
\hline 7 & $\begin{array}{l}\text { Este artículo es el aparte de una conferencia. Trata acerca del desarrollo de una plataforma llamada } \\
\text { Mediktor, que a través de una APP desarrollada con conceptos de inteligencia artificial, permite } \\
\text { que el estudiante de medicina se acerca más a los conceptos necesarios para realizar4diagnósti- } \\
\text { cos acertados dado que la herramienta facilita este fin, que a su vez alimenta una base de datos } \\
\text { mundial que a largo plazo, a través de big data, se espera poder explotar para otorgar mejores } \\
\text { diagnósticos. }\end{array}$ \\
\hline 8 & $\begin{array}{l}\text { El artículo realiza una revisión sistemática sobre la utilización de juegos en el proceso de ense- } \\
\text { ñanza aprendizaje en experiencias del área de la salud. Se contrasta la información encontrada en } \\
11 \text { artículos relevantes con el fin de determinar que la gamificación hace aportes a la experiencia } \\
\text { en clínica, simulación de condiciones en pacientes o virtualización de curso para la demanda de } \\
\text { rotaciones de los estudiantes de dichas áreas. }\end{array}$ \\
\hline 9 & $\begin{array}{l}\text { El artículo muestra la experiencia de apropiación de un software educativo fundamentado en la } \\
\text { gamificación para el área de la salud. Esta experiencia es probada por un grupo de estudiantes } \\
\text { residentes de medicina de la universidad de Alabama. El juego se llama Kaizen internal medicine } \\
\text { y busca reforzar conceptos, además de verificar en el tiempo la apropiación de los mismos. El } \\
\text { artículo muestra los resultados de la implementación del juego y cómo este ha sido aceptado entre } \\
\text { el público objetivo. }\end{array}$ \\
\hline
\end{tabular}




\begin{tabular}{|c|c|}
\hline$I D$ & Sintesis del artículo \\
\hline 10 & $\begin{array}{l}\text { Artículo de conferencia que busca ahondar sobre el IBL (internet based learning) utilizado en las } \\
\text { áreas de la salud para sus procesos de enseñanza aprendizaje y cómo la gamificación ha ayudado } \\
\text { a que este proceso se muestre más efectivo. Revisa literatura relevante en el tema y concluye que } \\
\text { las características de fidelización e interés que despierta la gamificación hace más efectivo el } \\
\text { proceso de enseñanza aprendizaje. }\end{array}$ \\
\hline 11 & $\begin{array}{l}\text { Explica cómo la gamificación y las reglas basadas en metodologías de diseño permiten la generación } \\
\text { de conocimiento y el aprendizaje profundo en el diseño arquitectónico. Por medio de una plataforma de } \\
\text { diseño gamified participan varios actores y generan un diseño colaborativo de vivienda masa urbana. } \\
\text { En este trabajo se demuestra la demanda de un siguiente paso en la investigación para crear } \\
\text { un sistema integrado que permita una mejor comunicación entre los usuarios y también con el } \\
\text { arquitecto principal, que podría promover la formación de las comunidades durante el proceso. } \\
\text { El diseño de elementos con gamificación juega un papel importante en la creación de una experiencia } \\
\text { atractiva que es el objetivo de este proyecto. }\end{array}$ \\
\hline 12 & $\begin{array}{l}\text { Artículo que evalúa la efectividad del aprendizaje del lenguaje C y el compromiso de los estudiantes } \\
\text { mediante la gamificación. Se utiliza un método exploratorio secuencial mixto el cual brinda resulta- } \\
\text { dos positivos en el empoderamiento de los estudiantes con las actividades de aprendizaje por medio } \\
\text { de la gamificación. Se creó la plataforma Q-learning-G el cual generaba la participación mediante } \\
\text { los sistemas de compensación o reconocimiento. Los resultados cuantitativos muestran dan la idea } \\
\text { que las actividades de aprendizaje combinadas con elementos del juego atren a los estudiantes para } \\
\text { alcanzar su propia meta, aprender. }\end{array}$ \\
\hline 13 & $\begin{array}{l}\text { Se centra el en desempeño de los cursos con estrategias de gamificación. El artículo evalúa cómo } \\
\text { la gamificación afecta a estudiantes de un curso de maestría semestral anual en Sistemas de infor- } \\
\text { mación e Ingeniería Informática en el Instituto Técnico superior de un curso llamado producción } \\
\text { de contenidos Multimedia (MCP). Los estudiantes encontraron el curso motivador e interesante, } \\
\text { aunque admiten que requiere más trabajo. Esto sugiere que los estudiantes se sientan más compro- } \\
\text { metidos con los cursos que utilizan gamificación, incluso si eso se traduce en una mayor carga de } \\
\text { trabajo. El enfoque de la investigación destaca que la gamificación, tiene potencial para animar a } \\
\text { los estudiantes a ser más diligente y dedicado al curso. }\end{array}$ \\
\hline 14 & $\begin{array}{l}\text { El artículo presenta algunas iniciativas desarrolladas en la Universidad de Alicante donde se } \\
\text { incorporaron videojuegos a la actividad académica e investigativa fruto de los proyectos finales } \\
\text { desarrollados en la carrera de Ingeniería en informática. }\end{array}$ \\
\hline 15 & $\begin{array}{l}\text { Artículo que cuenta la experiencia de ludificar un curso de administración y los resultados obtenidos } \\
\text { en. Para ludificar un curso incorporando juegos serios y simuladores, o de plano gamificándolo, } \\
\text { se puede llegar a asumir que el énfasis está en las herramientas, técnicas y dinámicas; pero el alma } \\
\text { del proceso educativo está en la relación que el profesor establece con los alumnos. Los niveles de } \\
\text { involucramiento e interés de los alumnos tienden a ser muy altos. }\end{array}$ \\
\hline 16 & $\begin{array}{l}\text { Este artículo tiene como objetivo evidenciar los efectos de la gamificación en un curso durante un } \\
\text { semestre académico. Comparando un curso gamificado y otro no. Para conseguir este objetivo, los } \\
\text { autores reunieron a } 80 \text { estudiantes en dos clases separadas de un mismo curso universitario. En los } \\
\text { cursos se ofrecieron los mismos materiales, tareas, exámenes y conferencias, con la diferencia de }\end{array}$ \\
\hline
\end{tabular}




\begin{tabular}{|c|l|}
\hline$I D$ & \multicolumn{1}{c|}{ Síntesis del artículo } \\
\hline 17 & $\begin{array}{l}\text { que uno tenía componentes metodológicos de la gamificación. Se plantea que la gamificación en } \\
\text { las aulas puede ser un "arma de doble filo" debido a que puede generan desmotivación en alumnos } \\
\text { que ya estaban motivados previamente. }\end{array}$ \\
\hline $\begin{array}{l}\text { Se revisa el caso de una implementación de un cursos gamificado de estudiantes de informática en } \\
\text { las asignaturas ingeniería de software y arquitectura orientada a servicios durante el } 2012 \text { y } 2013 . \\
\text { Se analiza el caso de un curso gamificado y otro no, en los cuales indicadores como asistencias, } \\
\text { grupo gamificado, no obstante la evaluación fue significativamente menor en éste. Aunque esto no } \\
\text { representa una conclusión mayoritaria si plantea la discusión al rededor de la pertinencia de imple- } \\
\text { mentar estrategias de éste tipo, profundizando en el análisis conceptual más allá de la herramienta. }\end{array}$ \\
\hline 18 & $\begin{array}{l}\text { El ejercicio consiste en la simulación del montaje de una serie de sets de lego, en los cuales son } \\
\text { trabajadas competencias básicas de la animación por computador como son iluminación, texturas y } \\
\text { modelado 3D. El artículo cuenta con una amplia contextualización alrededor de la gamificación, su } \\
\text { utilización y cómo ésta puede alinearse con los objetivos pedagógicos, sin embargo siendo un estudio } \\
\text { en curso sus fases de evaluación no se han realizado y los hallazgos reportados se encuentran sobre } \\
\text { la importancia de establecer una línea metodológica y conceptual en el planteamiento del juego. }\end{array}$ \\
\hline 19 & $\begin{array}{l}\text { El artículo presenta un resumen histórico de las perspectivas educativas en diferentes univeri- } \\
\text { sidades de australia y nueva zelanda, en donde los mundos virtuales (ambientes simulados con } \\
\text { diversos usos, incluida la gamificación) han tomado alta relevancia en los procesos formativos. El } \\
\text { desarrollo del estudio únicamente da indicios de en qué facultades se ha implementado y en qué } \\
\text { disciplinas, evidenciando que humanidades, letras, artes y cine tienen una baja recurrencia en el } \\
\text { total de programas que se referencian en el artículo, por ende pocas facultades usan la gamificación } \\
\text { para enseñar dichas disciplinas. }\end{array}$ \\
\hline
\end{tabular}

Fuente: elaboración autores

\section{EVALUACIÓN DE RESULTADOS-ANÁLISIS Y DISCUSIÓN DE RESULTADOS}

Para el análisis segmentado por área de conocimiento se representan los datos mediante la sumatoria de participación porcentual de los resultados de las cadenas de búsqueda en cada base de datos que en la revisión fue denominado como Score y se definió de la siguiente forma:

$$
\text { Score }_{i}=\sum_{j=1}^{9} P_{i, j} ; \forall i \in I
$$

Ecuación 1. Definición de variable: Score

$$
P_{i, j}=\frac{R_{i, j}}{\sum_{i=1}^{6} R_{i, j}} ; \forall j \in J
$$

Ecuación 2. Definición de variable: Porcentaje de participación 
Donde,

Score $_{i}$ : Sumatoria de participación porcentual de los resultados de las cadenas de búsqueda en cada base de datos

I: Conjunto de áreas de conocimiento, indexado con $i$ (seis áreas de conocimiento)

$J$ : Conjunto de bases de datos, indexado con $j$ (nueve bases de datos)

$P_{i, j}$ : Porcentaje de participación de los resultados del área de conocimiento i en el total de la base de datos $j$.

$R_{i, j}$ : Total de resultados para el conjunto de cadenas de búsqueda del área de conocimiento $i$ en la base de datos $j$.

A partir de los resultados, se realiza un análisis grupal de los datos obtenidos por área de conocimiento, como se puede observar en la figura 2. En este listado se indica el score para cada una:

1. Ingeniería y arquitectura - Score 3,15

2. Administración y economía - Score 2,46

3. Ciencias sociales y jurídicas - Score 1,31

4. Ciencias naturales y exactas - Score 1,07

5. Artes y humanidades - Score 0,53

6. Ciencias de la salud - Score 0,49

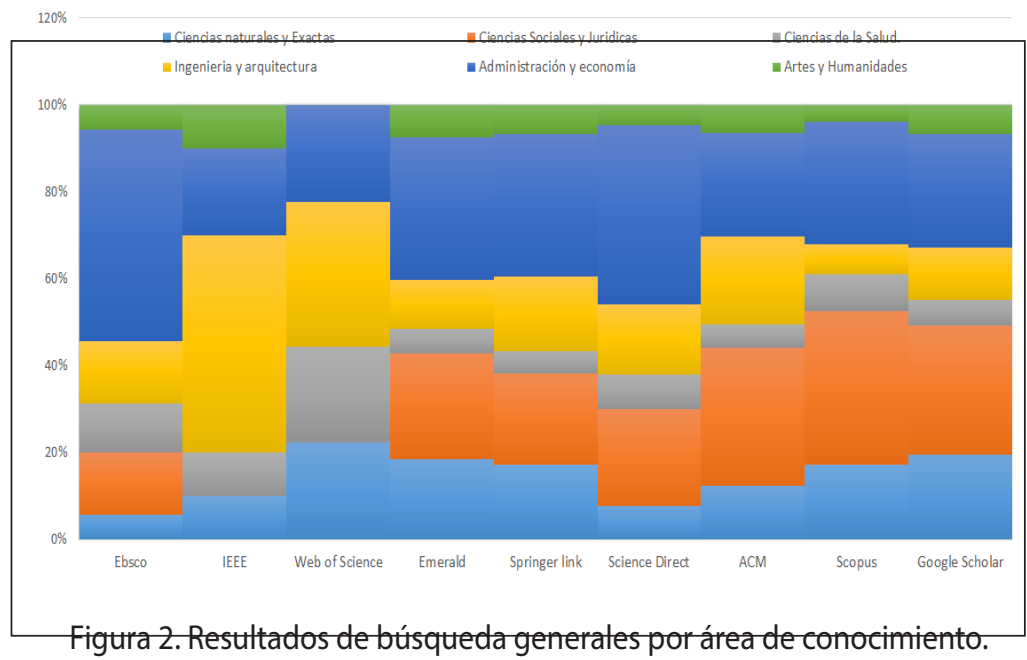

Fuente: elaboración autores

Por medio de un gráfico de áreas, se representa la dispersión de los resultados 
para cada área de conocimiento, mostrando la presencia de los términos rastreados en las ecuaciones de búsqueda. Esto da indicios de la dimensión del estado del arte de la gamificación en Educación Superior entre las áreas de conocimiento y su configuración. Los hallazgos indican que las áreas de Ingeniería y Arquitectura Administración y Economía y Ciencias Sociales y Jurídicas cuentan con mayor número de registros; esto se observa en la figura 2, donde la coloración azul oscuro, amarilla y naranja es más representativa que la azul claro, gris y verde, correspondientes a las áreas de conocimiento de Ciencias Naturales y Exactas, Ciencias de la Salud y Artes y Humanidades, respectivamente.

Para el análisis particular por área de conocimiento se consideran los datos obtenidos de cada ecuación y se realiza un procedimiento homólogo al análisis general, mostrando información por sus diferentes disciplinas. A continuación, se presentan unos casos de estudio, divididos según las áreas de conocimiento y teniendo en cuenta algunas de las disciplinas de las mismas.

\section{Ingeniería y arquitectura}

De acuerdo con los resultados obtenidos, la ingeniería y arquitectura son las que presentan una mayor participación en la producción de trabajos, con un score de 3,15, teniendo mayor desarrollo bibliográfico el área de ingeniería y con una amplia participación los programas de desarrollo de software e informática (ver figura 3 Ingeniería y Arquitectura). Esto puede ser un indicador importante sobre el papel que puede tener la tecnología en las metodologías de la gamificación.

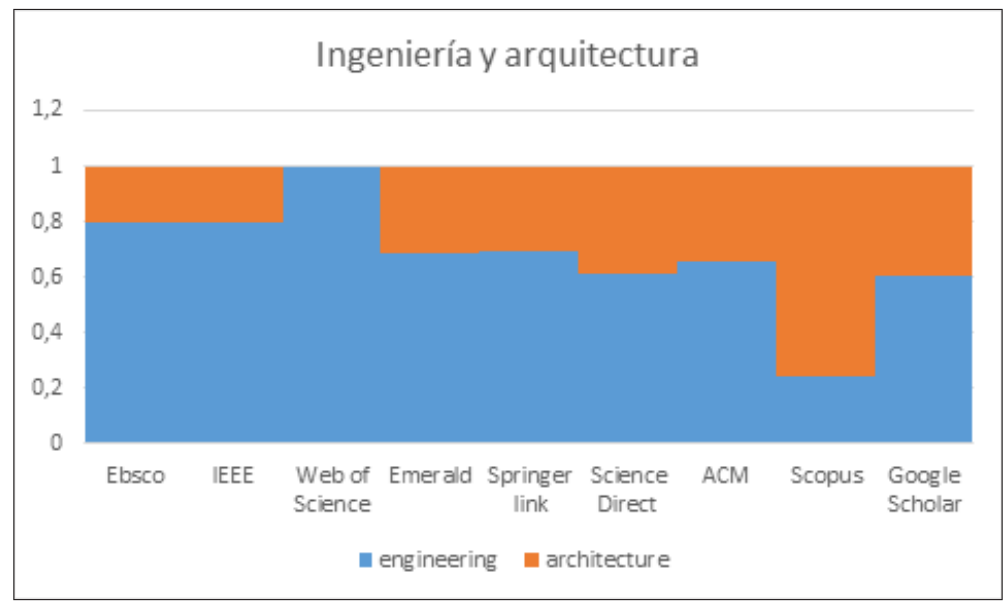

Figura 3. Ingeniería y Arquitectura

Fuente: elaboración autores 
El trabajo seleccionado para esta área es una experiencia significativa de la implementación de cursos gamificados para estudiantes de Informática en las asignaturas Ingeniería de Software y Arquitectura orientada a servicios durante los años 2011, 2012 y 2013. Aquí, el autor plantea la siguiente pregunta: ¿La inclusión de los elementos más básicos de la gamificación (tablero de puntos, liderazgo reconocido y factores de rivalidad) afecta el desempeño de los estudiantes? y para resolverla desarrolla dos experimentos: en el primero identifica tres grupos de Ingeniería de Software (15 estudiantes por grupo), uno gamificado y los otros dos como control, y compara los resultados de tres evaluaciones parciales y un trabajo final, además de una serie de tareas bonus en los cursos de los años en mención. En la experiencia, se encuentra que si bien en el grupo donde se implementó la estrategia de gamificación se presentan indicadores asociados a la motivación (como asistencia promedio y número tareas bonus realizadas) con un comportamiento mayor que en los grupos de control, estos últimos obtuvieron una diferencia significativa superior en la nota final promedio del curso, lo que mostró que, aunque los estudiantes se encontraron motivados por la temática, su desempeño académico fue inferior [8].

Ya en el segundo experimento, de cuatro grupos (15 estudiantes por grupo) del curso de arquitectura orientada al servicio, se gamifican dos. En este experimento el autor elimina el componente de rivalidad y define una escala de niveles en los cuales los estudiantes avanzan sin competir; además, incluye unos componentes de reconocimientos reales y tangibles por los puntajes simbolizados en fichas de póquer y medallas. Al igual que en el primer experimento, la motivación en asistencia y frecuencia de realización de trabajos aumenta en los grupos gamificados; de igual forma para este caso la calificación de los grupos que no contaron con dichos elementos es significativamente superior [8].

El autor analiza que, aunque con contundentes resultados en contra del desempeño académico, este es un estudio aislado, y concluir sobre la eficacia de la gamificación en la educación requiere análisis de mayor índole y contexto. No obstante, es interesante encontrar que al igual que muchos autores, este trabajo concluye que la gamificación como elemento motivador para el aprendizaje es un aliado de los procesos formativos, permitiendo romper barreras geográficas por la facilidad de virtualización que tiene; sin embargo, la inclusión de estas técnicas y herramientas en los procesos de educación debe analizarse de manera más profunda, dado que la utilización de nuevas tecnologías no es la excusa de un detrimento en la calidad de la educación.

\section{Administración y economía}

Al nivel organizacional, la gamificación se ha aplicado a Ventas o Marketing para la fidelización de clientes, pero comienza a emplearse de forma creciente en el aprendi- 
zaje y en la formación de estas áreas de conocimiento (ver figura 4 Administración y Economía).

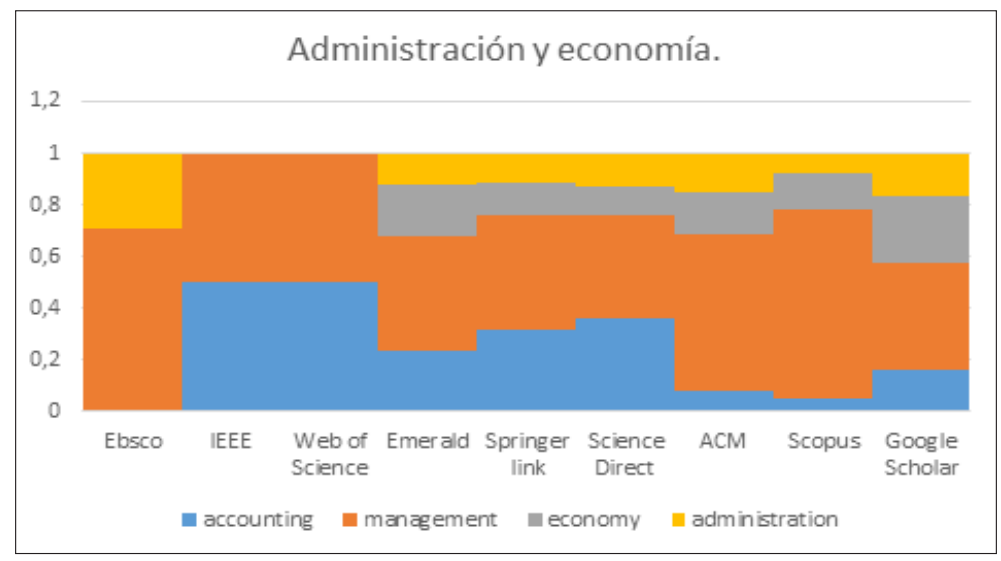

Figura 4. Administración y Economía

Fuente: elaboración autores

Los espacios gamificados no son un juego, sino experiencias que aplican elementos, mecánicas y dinámicas de este para fomentar el involucramiento de los participantes. En este sentido, en el artículo Experiencias de aprendizaje significativo mediante ludificación en un curso de administración, [9] buscaron promover mayores niveles de compromiso, involucramiento, motivación e inspiración en los estudiantes de Administración, y trasferir de manera natural los aprendizajes del mundo laboral al educativo. En el mundo laboral se vienen dando reflexiones y generando intervenciones para lograr altos niveles de involucramiento y compromiso tanto en clientes como en empleados. La estrategia partió de los objetivos del aprendizaje. Con las intenciones del curso se generó un enfoque llamado "nace un mánager"; en este los alumnos van escalando la jerarquía de la empresa haciendo méritos representados por los puntos de experiencia acumulados.

Los resultados indican que se presentan altos grados de involucramiento y entusiasmo. Hay alumnos que realizan muchas más actividades de las esperadas, alcanzando los mil puntos un mes antes de terminar el curso y continuando hasta acumular $1600 \mathrm{o}$ 1800 puntos. Algunas de las metodologías usadas permitieron a los estudiantes a través de la práctica afianzar el conocimiento. Otro aspecto para resaltar fue el replantear la participación del profesor, no enfocada en dominar el escenario, sino en acompañar y motivar al alumno al autoaprendizaje, evitando exposiciones largas de los temas y enfocándolas más a intervenciones cortas. Gamificar un curso requiere de mucho tiempo, ya que se debe pensar muy bien su contenido, las actividades de aprendizaje 
y los recursos de apoyo de clase. Además, no pueden ser cursos lineales; deben ser incrementales para que generen mayo enganche en los alumnos.

\section{Ciencias Sociales y Jurídicas}

Según los resultados, esta área de conocimiento cuenta con mayores trabajos en psicología, derecho e historia. Para conocer cómo se ha trabajado, abordamos el artículo de los autores en [10], Assessing the effects of gamification in the classroom: A longitudinal study on intrinsic motivation, social comparison, satisfaction, effort, and academic performance. Este estudio tuvo como objetivo evidenciar los efectos de la gamificación en un entorno real y con espacio de tiempo, que les permitiera tener medidas y la comparación con un entorno de clase no gamificado. Para alcanzar este objetivo, los autores reunieron a 80 estudiantes en dos clases separadas de un mismo curso universitario. En los cursos se ofrecieron los mismos materiales, tareas, exámenes y conferencias, con la diferencia de que uno tenía componentes metodológicos de la gamificación como tablas de clasificación, insignias, y sistemas de incentivos.

Se recogieron datos cuatro veces por separado en el transcurso de 16 semanas de un semestre y se tuvieron en cuenta variables motivacionales, psicológicas, así como variables de comportamiento. De los resultados obtenidos, los autores en su estudio plantean que la gamificación en las aulas puede ser un "arma de doble filo" debido a que puede generar desmotivación en alumnos que ya estaban motivados internamente, sin necesitar estrategias o de este tipo de metodologías. Sugiere que los profesores deben tener cuidado porque la "diversión obligatoria" puede ser más efectiva cuando el estudiante puede elegir o no participar de la misma. Además de esto, también se identificó que el compromiso y el interés disminuyeron con el tiempo para los estudiantes del curso gamificado (ver figura 5 Ciencias Sociales y Jurídicas).

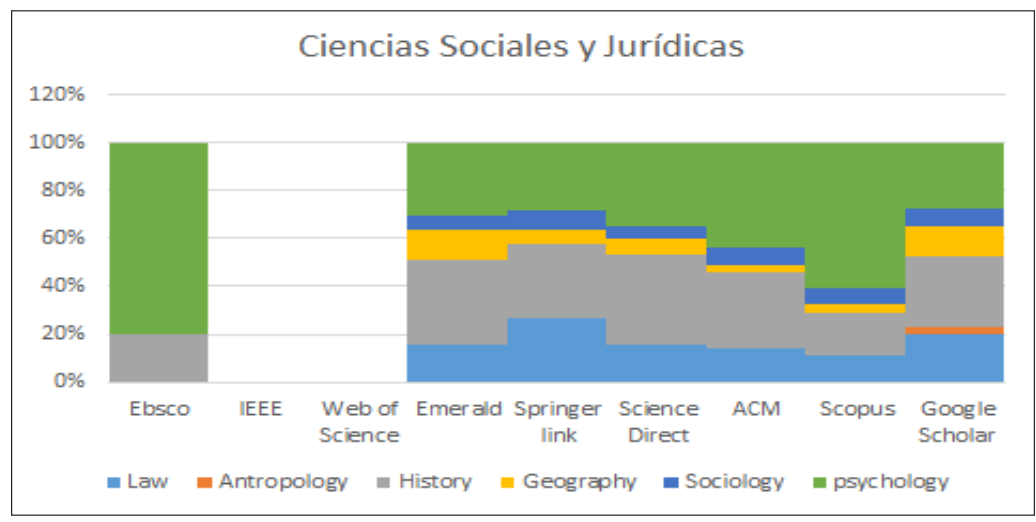

Figura 5. Ciencias Sociales y Jurídicas.

Fuente: elaboración autores 


\section{ciencias Naturales y Exactas}

El área de las ciencias naturales, según los resultados, se ubica en cuarto lugar con una participación importante en las disciplinas de matemáticas, física, química en dichas áreas (ver figura 6 Ciencias Naturales y Exactas).

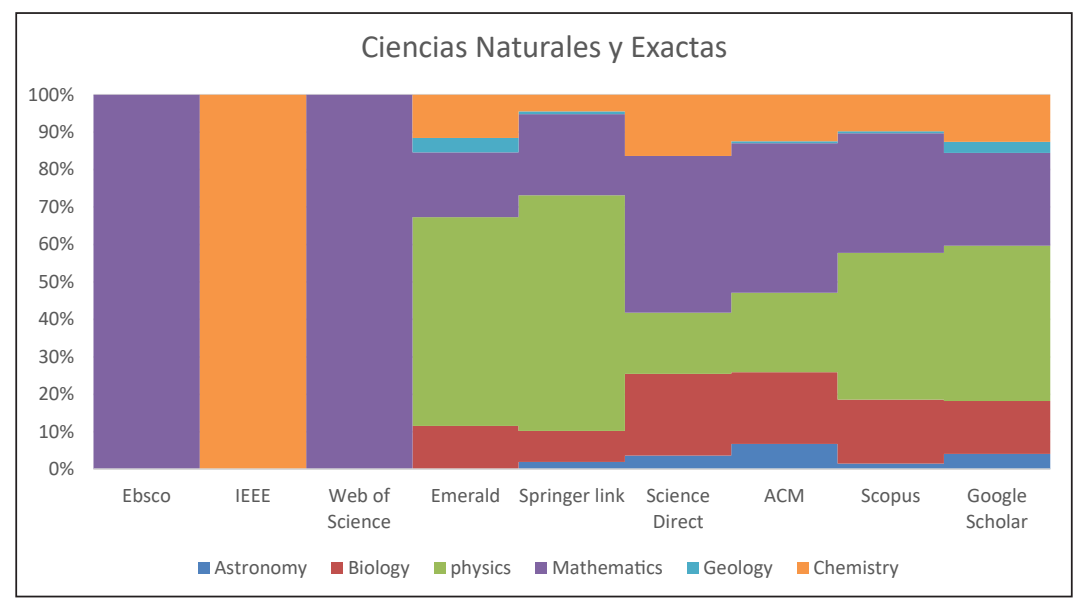

Figura 6. Ciencias Naturales y Exactas.

Fuente: Elaboración autores

Para conocer cómo ha sido abordada la gamificación en este caso, se presenta el artículo Improving biotech education through gamified laboratory simulations [11] el cual muestra la implementación de esta metodología para la docencia en los laboratorios, específicamente mediante la simulación de los mismos, en el cual se puedan vencer barreras como costo, tiempo, seguridad de los alumnos y, su vez, motivar el aprendizaje de los estudiantes. Según el artículo, los simuladores están dando posibilidades de aprendizaje a los estudiantes, logrando reconocer, por ejemplo, partículas imperceptibles para el ojo humano y comportamientos no vistos al alcance de una enseñanza en laboratorio tradicional. En este trabajo, los autores no solo identifican la simulación como una alternativa, sino que reconocen la tecnología como un gran aliado para el aprendizaje que se debe aprovechar. Es por esto que en el estudio de caso, se desarrolló y probó una plataforma-laboratorio basado en algoritmos matemáticos de investigación de apoyo-abierto y elementos de gamification como un universo 3D, narración de cuentos, conversaciones con personas de ficción y un sistema de puntuación. El resultado fue un avance del $76 \%$ en el aprendizaje mediante el uso de la simulación de laboratorio en comparación con una enseñanza tradicional; y más del 100\% cuando se combinan las formas de enseñanza. Se espera que se realicen más estudios para la enseñanza con simulaciones en el área de biotecnología, donde se ha utilizado poco y la cual fue objeto de estudio. Asimismo, el trabajo recomienda establecer una ten- 
dencia general en la eficacia de las simulaciones y generar otras formas de educación que vayan más allá de los métodos tradicionales de la enseñanza en los laboratorios.

\section{Artes y humanidades}

De acuerdo con los registros de estudios publicados, no se presentan muchos casos en esta área de conocimiento. El artículo presentado para la revisión se titula "Gamification for Learning 3D Computer Graphics Arts" y es una aplicación del concepto para la enseñanza en un pregrado de multimedia en la disciplina de Animación por Computadora. El estudio en curso tiene como finalidad encontrar los efectos que tiene en los estudiantes la inclusión de este tipo de estrategias; por ende, se proponen dos tipos de pruebas durante el trabajo: unas encaminadas a evaluar variables objetivas de desempeño y metas de los jugadores, y otras planteadas desde teorías constructivistas del conocimiento. El ejercicio consiste en la simulación del montaje de una serie de sets de lego, en los cuales son trabajadas competencias básicas de la animación por computador como son iluminación, texturas y modelado 3D, que dependiendo de la cantidad de modelos en los cuales los estudiantes trabajan, y la complejidad de los mismos, reciben puntos lo que realimenta la experiencia al resto del grupo y genera ambiente de competencia. El artículo cuenta con una amplia contextualización alrededor de la gamificación, su utilización y cómo esta puede alinearse con los objetivos pedagógicos; sin embargo, siendo un estudio en curso sus fases de evaluación no se han realizado y los hallazgos reportados se encuentran sobre la importancia de establecer una línea metodológica y conceptual en el planteamiento del juego [12]. (Figura 7 Arte y humanidades)

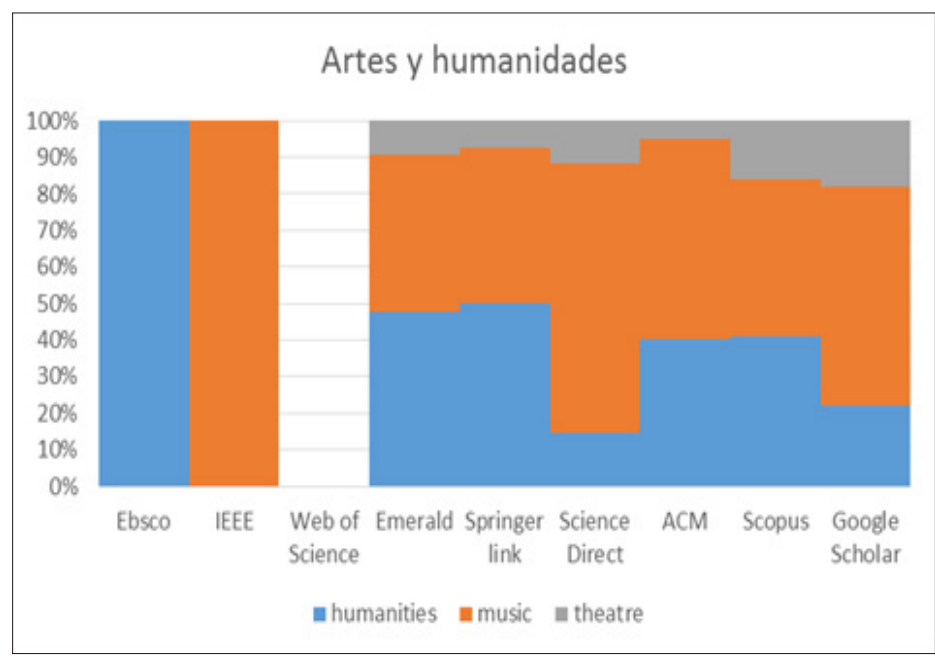

Figura 7. Arte y humanidades

Fuente: elaboración autores 
La gamificación en ciencias de la salud ha sido abordada desde sus diferentes áreas específicas con implementaciones y estudios, que dan cuenta de la utilidad de este tipo de estrategias para mejorar la motivación de los estudiantes al abordar los conceptos y que permiten, a su vez, sortear desafíos educacionales (Ver figura 8 Ciencias de la salud).

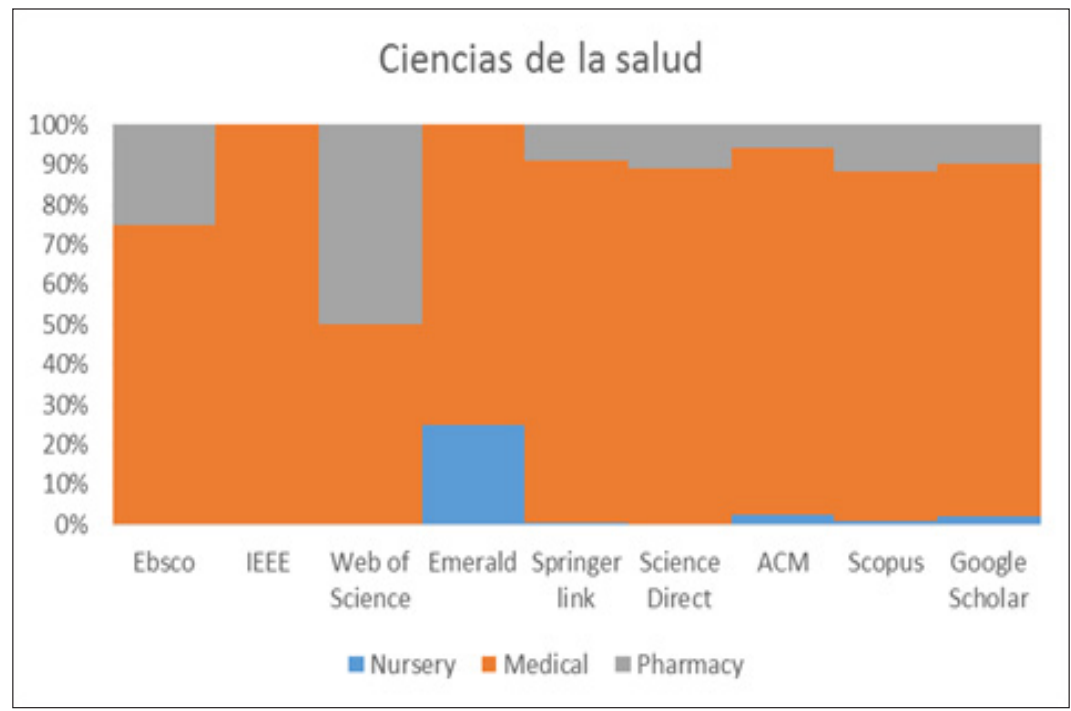

Figura 8. Ciencias de la salud.

Fuente: elaboración autores

Así lo muestra [13] quien realiza una revisión sistemática sobre la utilización de juegos en el proceso de enseñanza-aprendizaje en el área de la salud. A través de un proceso de búsqueda, depuración, evaluación y selección, se contrasta la información encontrada en 11 artículos relevantes con el fin de determinar factores comunes y beneficios que ha brindado la utilización del concepto.

Los diferentes enfoques encontrados en la revisión son aplicaciones basadas en TIC, experimentos con maniquíes para el estudio de disciplinas como la anatomía o puzles para memorizar conceptos técnicos. Estos muestran resultados en la motivación de los estudiantes al abordar nuevos conceptos en ambientes interactivos de colaboración o competencia; sin embargo, entre las barreras encontradas para este tipo de estrategias se encuentra el costo de desarrollo de aplicativos y la dificultad de concluir acerca de los efectos positivos que la utilización de la gamificación tiene en el aprendizaje en comparación con otros métodos. Los altos costos de desarrollo se ven compensados con la escalabilidad que tienen este tipo de estrategias en situaciones que requieren alta cobertura como la educación virtual o semipresencial. 
Finalmente, el autor concluye que la utilización de la gamificación en las disciplinas que competen a las ciencias de la salud brinda herramientas de entrenamiento a los estudiantes los cuales pueden prepararse para una situación real mediante experiencias simuladas. Desde el punto de vista del desarrollo de los juegos, estos pueden llegar a ser costosos y complejos de llevar a cabo, por lo que deben ser planeados con mucha cautela para que no representen una pérdida de tiempo ni de dinero en el desarrollo.

\section{DISCUSIÓN}

Los resultados indican que el área de conocimiento de Ingeniería y arquitectura (Score 3,15 ) arroja los registros más altos, sucedida por Administración y economía (Score 2,46). Los trabajos revisados en estas áreas y sus disciplinas, muestran la implementación de elementos comunes de gamificación como: simulación, puntajes, escalas de liderazgo, entre otros [8], [9]. En Ciencias sociales y jurídicas (Score 1,31), y Ciencias naturales y exactas (Score 1,07), la gamificación es aplicada con distintos elementos mostrando un resultado positivo en las ciencias naturales, pero no tan favorable para el caso de estudio de las ciencias sociales [10], [11]. Finalmente en las áreas de Artes y humanidades (Score 0,53) y Ciencias de la salud (Score 0,49), aunque con menor representación en el total de búsquedas, igualmente se presentan experiencias significativas [12], [13] en los trabajos revisados, especialmente con aplicación de la gamificación como herramienta de práctica para los estudiantes.

Entre los elementos de los estudios se encuentra que la función de la gamificación en algunos casos se aplicó como complemento a procesos de enseñanza tradicionales y en otros cursos totalmente gamificados. En ambos casos, es necesario establecer los objetivos de aprendizaje y dirigir los componentes del juego hacia ello [9], [11], [14]. De igual manera, es importante, al definir un juego, considerar cuál es el área específica de conocimiento, la complejidad de la disciplina y los perfiles de los jugadores de modo que se obtenga un efecto positivo y no se desvirtúe su objetivo inicial.

Frente a los resultados de la gamificación en la enseñanza, particularmente en las calificaciones, los trabajos revisados no concluyen de forma unificada: en ciertos casos no hay una medición que dé cuenta por la mejora en el desempeño [9], [14] y en otros casos [9-10] se cuantifican efectos no satisfactorios [8], [10]. No obstante, considerando que en la revisión no se encontró que sobre la temática en la Educación Superior se cuente con estándares, se requiere contar con un trabajo que recoja estas experiencias para su comparación y generar posibles tendencias del tema.

Sin duda alguna, la tecnología es una aliada para la gamificación, de forma tal que mediante su uso se pueden desarrollar ambientes simulados [11] y herramientas web 2.0 que faciliten la comunicación, el carácter social que propicie la interacción [3] y 
facilite la apropiación de las herramientas. Sin embargo, es importante mencionar que la gamificación no solo está asociada a la tecnología y no es en sí misma informática.

\section{SOLUCIÓN PROPUESTA}

A partir de la revisión de literatura sobre gamificación en la Educación Superior, se logra evidenciar que este tema aún es novedoso y en algunos casos poco explorado en las diferentes áreas de conocimiento. Actualmente no se cuenta con una teoría unificada o un conjunto de lineamientos que permitan ver el concepto de manera holística y mucho menos cuando se trata de aplicarlo en la Educación. En los trabajos revisados, el término se aborda de forma indiscriminada y no permite con claridad distinguir si es una técnica, una metodología, una estrategia o una dinámica. Esto a futuro plantea inquietudes como: ¿Cómo medir el impacto o efectos de la gamificación, teniendo en cuenta que no hay un concepto unificado o estándar asociado a ella? ¿Más allá de la motivación, cómo puede la gamificación influir en el desempeño académico del alumno en la Universidad? ¿Es apropiado aplicar la gamificación en todas las áreas de conocimiento?

Con el interés de dar respuesta a estos y otros interrogantes, como solución de esta revisión, a continuación se expone una serie de recomendaciones sobre la gamificación en la Educación Superior, como una reflexión sobre lo que se puede mejorar y potenciar en este campo:

- Es importante definir objetivos puntuales en la implementación de la gamificación en la Educación Superior y los efectos que esta genera en la enseñanza-aprendizaje. Una manera de construir o llegar a estos es contar con un número de experiencias significativas, con indicadores y demás que permitan comparar distintas estrategias empleadas. Hasta el momento lo que se encuentra son experiencias aisladas y sin oportunidad de comparar los resultados obtenidos en diferentes instituciones, países, etc. De igual forma, en los trabajos revisados se evidenció que las investigaciones por lo general se hacen con muestras muy pequeñas y en tiempos muy cortos, lo cual se considera poco apropiado en el ámbito educativo, donde se puede requerir comparar muestras más grandes y lapsos de tiempos mucho más amplios.

- A futuro, sería interesante contar con estudios por áreas de conocimiento sobre la implementación y resultados de la gamificación. Que se logre unificar criterios de cómo y en qué ámbitos de los diferentes saberes es viable emplearla con sus distintas metodologías. Se deberá tener en cuenta entonces que los juegos sean acordes a las áreas de conocimiento y se asegure realmente un resultado favorable. En este sentido, es de vital importancia la precaución frente a las propuestas de 
estrategias de juego, las cuales no terminen afectando los intereses o motivaciones para el aprendizaje de los alumnos de una manera negativa.

- Teniendo en cuenta que la gamificación es un tema novedoso y que está comenzando a generar mayor interés en el ámbito de la Educación Superior, es importante realizar un constante seguimiento sobre nuevos trabajos y temáticas que surgen de la misma, e identificar los avances en cada una de las áreas de conocimiento y los distintos contextos en los que se están aplicando. La aplicación de nuevas y más detalladas ecuaciones de búsqueda facilitaría realizar el ejercicio de vigilancia constante.

\section{CONCLUSIONES}

Como lo muestra la revisión, los juegos en ambientes no lúdicos están ganando espacio en la Educación. El interés de conocer cómo se estaba abordando esta novedosa temática en la Educación Superior orientó la revisión a las diferentes áreas de conocimiento y disciplinas, intentando conocer más sobre su aplicabilidad

En los estudios seleccionados, se identificaron factores comunes y diferencias, encontrando que la gamificación puede tener impactos positivos y negativos en la motivación e interés de los alumnos, al igual que en sus desempeños académicos. Por esto, se considera necesario que se pueda hablar de un marco común o de unos lineamientos claros de aplicación de la misma, especialmente identificar qué puede ser apropiado para las distintas áreas de conocimiento, bien sea para aplicarlo en la Educación Superior o en ámbitos organizacionales.

Según la revisión, las áreas de Ingeniería-Arquitectura, Administración-Economía y Ciencias Sociales-Jurídicas cuentan con mayor número de registros en sus cadenas de búsqueda, con lo cual se puede inferir que la gamificación ha generado mayor interés en estas áreas de conocimiento y se han motivado las investigaciones alrededor de su uso en la Educación Superior. Uno de los indicios más claros frente a los resultados puede estar en la influencia de la tecnología en el área de ingeniería. Una novedad pueden ser las ciencias sociales y jurídicas, que presentaron mayor número de registros en comparación con las ciencias naturales-exactas, donde se podría pensar tendría más campo de acción la gamificación, con las metodologías de juego para el aprendizaje. Por último, se encuentran las ciencias de la salud y artes-humanidades, donde los resultados de investigaciones no arrojaron datos representativos. Uno de los aspectos positivos de la revisión es lograr identificar trabajos para todas las áreas de conocimiento propuestas, lo que indica que activamente (aunque con diferente intensidad) la gamificación es una apuesta de las universidades en sus carreras. 
Es claro que lo relacionado con la gamificación es aún novedoso; sin embargo, se espera que siga teniendo mayor presencia en las investigaciones de Educación Superior y que se generen más estudios para la interacción enseñanza-aprendizaje. Por esto, es interesante seguir haciendo un seguimiento frente a la evolución que presenta la temática en un futuro próximo, sobre todo, en lo relacionado con su efecto y resultados frente a su uso y aplicación.

\section{AGRADECIMIENTOS}

A la profesora María Clara Gómez Álvarez como asesora temática y al profesor Jaime Alberto Echeverri, asesor metodológico.

\section{REFERENCIAS}

[1] E. Castañeda, C. Castañeda, S. Betancur, and J. M. G. Giraldo, "Desarrollo de un videojuego para la enseñanza del concepto de tragedia de los comunes en cursos de ingeniería", Rev. Educ. en Ing., vol. 8, n. ${ }^{\circ}$ 16, pp. 25-37, 2013.

[2] M. Durán Rodríguez, "Una renovación en el proceso de enseñanza y aprendizaje", Gestiopolis, 2010. [Online]. Available: http://www.gestiopolis.com/una-renovacion-proceso-ensenanzaaprendizaje/. [Accessed: 13-Oct-2016].

[3] R. N. Landers and R. C. Callan, "Casual social games as serious games: The psychology of gamification in undergraduate education and employee training", in Serious games and edutainment applications, Springer, 2011, pp. 399-423.

[4] J. J. Lee and J. Hammer, "Gamification in education: What, how, why bother?", Acad. Exch. $Q$. , vol. 15, n. ${ }^{\circ}$, p. 146, 2011.

[5] B. Marne, J. Wisdom, B. Huynh-Kim-Bang, and J.-M. Labat, "The six facets of serious game design: a methodology enhanced by our design pattern library" in European Conference on Technology Enhanced Learning, 2012, pp. 208-221.

[6] Observatorio laboral para la educación. Graduados Colombia, "área de conocimiento," Glosario, 2013. [Online]. Available: http://www.graduadoscolombia.edu.co/html/1732/ propertyvalue-37268.html. [Accessed: 13-Oct-2016].

[7] D. A. Morales Valencia, "Revisión sistemática a la literatura para determinar los enfoques de la investigación en verificación formal del software", 2012.

[8] M. Laskowski and A. Definition, "Implementing gamification techniques into university study path - a case study", 2015. IEEE Global Engineering Education Conference (EDUCON). March, pp. 582-586, 2015.

[9] E. B. Rangel, F. M. del Campo Sepúlveda, and G. I. Castanedo, "Experiencias de aprendizaje significativo mediante ludificación en un curso de administración", in ler Congreso Internacional de Innovación Educativa, 2014. 
[10] M. D. Hanus and J. Fox, "Assessing the effects of gamification in the classroom: A longitudinal study on intrinsic motivation, social comparison, satisfaction, effort, and academic performance", Comput. Educ., vol. 80, pp. 152-161, 2015.

[11] M. T. Bonde, G. Makransky, J. Wandall, M. V Larsen, M. Morsing, H. Jarmer, and M. O. A. Sommer, "Improving biotech education through gamified laboratory simulations," Nat. Biotechnol., vol. 32, n. ${ }^{\circ}$, pp. 694-697, 2014.

[12] S. Villagrasa and J. Duran, "Gamification for learning 3D computer graphics arts", in Proceedings of the First International Conference on technological ecosystem for enhancing multiculturality, 2013, pp. 429-433.

[13] M. S. Shawaqfeh, "Gamification as a Learning Method in Pharmacy Education," J. Pharm. Care Heal. Syst., vol. 2015, 2015.

[14] G. Barata, S. Gama, J. Jorge, and D. Gonçalves, "Engaging engineering students with gamification," in Games and Virtual Worlds for Serious Applications (VS-GAMES), 2013 5th International Conference on, 2013, pp. 1-8. 